\title{
Filigrane
}

Écoutes psychanalytiques

\section{La clinique de l'extrême dans le cadre de l'intervention humanitaire : le paradoxe du contenant non contenu}

\section{David Smolak et Louis Brunet}

Volume 28, numéro 2, 2019

URI : https://id.erudit.org/iderudit/1069700ar

DOI : https://doi.org/10.7202/1069700ar

Aller au sommaire du numéro

Éditeur(s)

Santé mentale et société

ISSN

1192-1412 (imprimé)

1911-4656 (numérique)

Découvrir la revue

Citer cet article

Smolak, D. \& Brunet, L. (2019). La clinique de l'extrême dans le cadre de l'intervention humanitaire : le paradoxe du contenant non contenu. Filigrane, 28(2), 189-203. https://doi.org/10.7202/1069700ar
Résumé de l'article

Les aidants humanitaires oeuvrent dans des contextes instables qui mettent à l'épreuve leur subjectivité. Ils sont confrontés à des conditions extrêmes de travail et développent des relations avec leurs patients qui sont chargées quantitativement et empreintes de contenus peu symbolisés. En réaction à cette expérience au haut potentiel traumatique, ils érigent des systèmes défensifs variés (expulsion de la quantité, coupure de soi, etc.) et présentent une diversité de symptômes plus ou moins couteux pour l'appareil psychique. En lien avec leur réponse à la situation extrême s'élabore aussi une demande de prise en charge des besoins de leur Moi à laquelle l'objet-environnement répond de façon plus ou moins ajustée. Ainsi, cet article porte un regard psychanalytique sur la clinique de l'extrême et propose divers dispositifs métacontenants qui pourraient agir comme des facteurs de protection pour les aidants. 


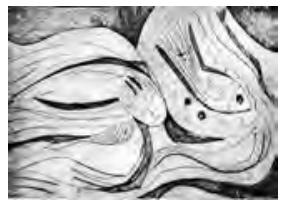

\title{
La clinique de l'extrême dans le cadre de l'intervention humanitaire: le paradoxe du contenant non contenu
}

\author{
David Smolak et Louis Brunet
}

\begin{abstract}
Résumé: Les aidants humanitaires ouvrent dans des contextes instables qui mettent à l'épreuve leur subjectivité. Ils sont confrontés à des conditions extrêmes de travail et développent des relations avec leurs patients qui sont chargées quantitativement et empreintes de contenus peu symbolisés. En réaction à cette expérience au haut potentiel traumatique, ils érigent des systèmes défensifs variés (expulsion de la quantité, coupure de soi, etc.) et présentent une diversité de symptômes plus ou moins couteux pour l'appareil psychique. En lien avec leur réponse à la situation extrême s'élabore aussi une demande de prise en charge des besoins de leur Moi à laquelle l'objet-environnement répond de façon plus ou moins ajustée. Ainsi, cet article porte un regard psychanalytique sur la clinique de l'extrême et propose divers dispositifs métacontenants qui pourraient agir comme des facteurs de protection pour les aidants.
\end{abstract}

Mots clés: traumatisme secondaire; traumatisme vicariant; clinique de l'extrême; survivance psychique; aide humanitaire.

\begin{abstract}
Humanitarian caregivers work in unstable contexts that challenge their subjectivity. They are confronted with extreme working conditions and develop relationships with their patients which are quantitatively intense and imprinted with unsymbolized contents. In response to this experience of high traumatic potential, they erect various defensive systems (expulsion of quantity, "cutting" away from themselves, etc.) and present a variety of symptoms more or less expensive for the psyche. In connection to their response to the extreme situation, they also express a demand or a need for the support of their ego to which the object-environment responds in a more or less adjusted way. Thus, this article proposes a psychoanalytical look at the clinic of the extreme, and suggests metacontaining modalities that could act as protective factors for caregivers.
\end{abstract}

Keywords: secondary trauma; vicarious trauma; psychic survival; clinic of the extreme; humanitarian aid. 
haque année, des centaines d'aidants humanitaires sont envoyés en mission dans des contextes extrêmes et désubjectivants. Selon certaines conceptions théoriques (voir Smolak et Brunet, 2017), ces soignants sont appelés à remplir une fonction de contenance, c'est-à-dire qu'ils tentent de comprendre et de tolérer le trop-plein d'angoisse de leurs patients, tout en transformant en contenus intelligibles et ré-intégrables leurs éprouvés mal symbolisés ou énigmatiques (Bion, 1962; Brunet et Casoni, 2000). Or, dans le cadre de leur travail, ils doivent non seulement contenir les vécus traumatiques de ceux qu'ils soignent, mais aussi traiter leurs propres réactions de sidération ou de débordement. Ainsi, l'intervention en contexte humanitaire ou auprès de victimes de traumatismes ébranle les soignants tant sur le plan personnel que sur celui de leurs fonctions thérapeutiques (voir par exemple Connorton, Hemenway et Miller, 2012; Quosh, Eloul et Ajlani, 2013). Leur exposition directe et indirecte à la souffrance traumatique met leur propre intégrité à rude épreuve, favorisant parfois pour eux-mêmes l'émergence d'un fonctionnement psychique «en traumatique ${ }^{1}$ » (Bokanowski, 2002, 2010).

L'espace réflexif accordé à l'expérience subjective des aidants humanitaires est minime dans le corpus scientifique d'aujourd'hui; les études actuelles se concentrent surtout à décrire les manifestations symptomatologiques «en positif» de la fatigue empathique et du traumatisme vicariant ou secondaire. Dans cet article, nous visons à exposer comment les soignants, en étant doublement exposés au traumatisme, en viennent à avoir eux-mêmes besoin de contenance et à développer des modes coûteux de survivance psychique (Roussillon, 2005). Nous décrivons, sous un angle psychanalytique, différents types d'aménagements psychiques qui émergent en fonction de la qualité de la réponse de l'environnement aux demandes de (méta) contenance des aidants rencontrés. Nous recommandons aussi des dispositifs pouvant agir comme des facteurs de protection pour l'intégrité psychique de tout soignant œuvrant dans un contexte extrême ou avec une population traumatisée.

Nous avons réalisé cinq entretiens d'une heure avec trois aidantes ayant prodigué ou prodiguant des soins en contexte humanitaire, totalisant quinze heures d'entrevue. Notre échantillon était limité, mais nous avons choisi de privilégier la profondeur plutôt que l'étendue afin de dépasser la simple description de leur expérience. Un devis de recherche qualitatif, basé sur l'analyse du discours, a été employé puisqu'il s'arrimait avec notre position épistémologique et nous permettait à la fois d'avoir accès au contenu manifeste 
et de faire des inférences quant au contenu latent. Les données recueillies ont été analysées dans un processus itératif d'analyse-retour en collaboration avec le second auteur de l'article; chaque entrevue était analysée en dyade avant de procéder à la suivante.

Dans le cadre de ce projet, nous avons rencontré: (1) Natalie ${ }^{2}$, infirmière ayant effectué deux missions humanitaires d'environ un an chacune, à Haïti, puis au Kosovo; (2) Marwa, psychologue œuvrant auprès de réfugiés de guerre, d'ex-prisonniers ou de victimes du régime d'oppression syrien; et (3) Thalia, elle aussi psychologue au Moyen-Orient auprès d'ex-prisonniers de guerre.

\section{Un contexte de soin doublement traumatique}

Les soignantes rencontrées ont dépeint un portrait chaotique et extrême du contexte dans lequel elles travaillaient. Chacune intervenait auprès d'une population polytraumatisée dans un cadre peu défini. Elles ne recevaient de la supervision que de façon sporadique ou grâce à leur propre initiative. Leur organisme d'appartenance n'offrait pratiquement pas de formations spécifiques aux enjeux inhérents à leurs rôles. Les aidantes se voyaient quotidiennement confrontées à la perte de patients ou de collègues. On leur demandait d'intervenir auprès de plus d'une dizaine de patients traumatisés par jour (réfugiés de guerre, ex-prisonniers, victimes de torture) en plus de recevoir et transformer l'expérience en attente de symbolisation des membres de leur équipe. Les soignantes s'offraient à leurs patients comme réceptacles de leurs souffrances traumatiques; elles se portaient implicitement garantes de la mise en sens des horreurs et de l'angoisse qui leur étaient partagées. Par l'entremise d'un jeu d'identifications projectives empathiques (Brunet et Casoni, 2000), elles faisaient l'expérience, en miroir, du vécu traumatique de leurs patients. Leur équilibre psychique était fortement taxé et leur disponibilité psychique mise en péril. Nous insistons sur le caractère doublement traumatique de leur expérience, et ce, en raison des circonstances déstructurantes dans lesquelles elles intervenaient, mais aussi de leur nécessaire empathie au vécu désubjectivant de leurs patients.

\section{Le besoin et la demande de contenance des aidantes}

Les aidantes ont exprimé le besoin que quelqu'un remplisse pour elles aussi une fonction de contenance, en réponse à cette double traumatisation. Elles souhaitaient faire la rencontre d'un objet disposé à recevoir et transformer les parties peu symbolisées de leur psyché et de leur expérience. 
Elles ont demandé à pouvoir jouir d'un espace où communiquer leur vécu traumatique afin qu'il soit traité et qu'il leur soit rendu sous une forme plus intégrée, tolérable et pensable (Bion, 1962; Brunet, 2013). Elles ressentaient le besoin que quelqu'un contienne leur propre trop-plein et sidération, pour y survivre et pour retrouver un certain sentiment de maitrise sur ceux-ci, mais aussi pour recouvrer leur fonction thérapeutique.

\section{Une demande de contenant}

Marwa raconte avoir fait l'expérience de la «sidération», de l'envahissement et de l'effacement de ses limites identitaires dans le cadre de son travail d'aide humanitaire. Elle rapporte avoir eu l'impression que «plus rien n'avait de sens» à la suite de certaines séances de thérapie traitant de contenus extrêmes ou horrifiques. Elle souligne avoir été bouleversée par le fait de côtoyer «la mort, tout le temps, tout le temps, tout le temps... la destruction» et précise que la question des sévices infligés aux prisonniers la touche particulièrement. "C'est quelque chose qui me sidère", ditelle, et qui devient même lourd et «agressant», surtout lorsque cela «[m'est partagé] hors cadre.» Marwa indique qu'il n'est pas rare que ses patients la contactent à l'extérieur de ses heures de travail (par exemple à $3 \mathrm{~h}$ du matin) pour lui faire part de leur souffrance et qu'ils résistent à ses tentatives de rétablissement du cadre thérapeutique. Souvent, cela la mène à devoir réitérer certaines limites et leur rappeler qu'elle n'est pas leur amie, mais bien leur psychologue. Le caractère extrême et «dramatique» de leur histoire participe aussi à la «confusion» identitaire perçue par l'aidante; Marwa a fait l'expérience de douleurs physiques ou de picotements dans son corps après des entretiens avec des patients ayant été victimes de torture. Pour ces raisons, elle mentionne avoir «besoin d'un cadre pour se protéger», en plus de «faire une séparation claire entre son travail et sa vie privée». Elle rapporte: «c'est comme si moi-même, après avoir écouté cette souffrance, j’ai besoin de la mettre quelque part, de la situer sur quelque chose». Or, elle se bute à l'absence d'espaces de contenance et d'élaboration institutionnalisés et se trouve à devoir créer ses propres réceptacles.

Marwa a contacté une association française spécialisée dans le traumatisme pour entreprendre une supervision individuelle d'approche psychanalytique. Elle déplore l'absence systématique d'espace de supervision dans ses milieux de travail. Elle rapporte: «Avec une des ONG avec qui je bosse, j'ai même exigé $[. .$.$] qu'on nous trouve une supervision pour le groupe,$ pour nous, l'équipe». Marwa se dit souvent «fatiguée [...] d'être à l'écoute 
de tout le monde» et ajoute qu'il est «relaxant de pouvoir évacuer [...] ce qui a été écouté, mais dans un champ professionnel où les collègues peuvent faire des commentaires». La soignante demande non seulement à pouvoir expulser le surplus d'excitation qui l'habite, mais elle cherche surtout à ce que cette quantité débordante puisse être reçue et transformée par un objet «qui comprend l'impact que ça peut avoir d'entendre des gens ayant subi divers traumatismes». L'appareil psychique de Marwa a été ébranlé par la surcharge quantitative et l'absurdité de son expérience. Or, sa psyché ne semble pas avoir été désorganisée au point de mettre en place un fonctionnement traumatique à proprement parler.

\section{Une demande d'objet}

Thalia relie sa demande de soutien à la «solitude de l'intervenant dans une situation impossible». Elle indique avoir éprouvé une forme particulière de solitude lors de ses expériences d'aide humanitaire, de par le fait d'être la seule psychologue de son équipe, mais aussi à cause de la nécessité de faire face, seule, à l'intensité et à l'horreur partagées par ses patients. "On n'a pas d'espace où partager», indique-t-elle. Lors de son expérience de soins en milieu hospitalier, elle rapporte: "Je n'avais pas de supervision et, pourtant, j'avais demandé [à en avoir].» Thalia n'arrivait plus à se «protéger [...] contre le burn out ou la traumatisation secondaire». Pour cette aidante, «ce manque de soutien était en soi traumatisant» et la menait à devoir porter à elle seule la «responsabilité» de métaboliser son expérience extrême en plus de celle de ses patients. Elle indique: «Comme pour le patient qui vit un trauma, les professionnels, eux aussi, ont besoin d'un soutien professionnel, c'est-à-dire un lieu de parole où ils peuvent exprimer tout ce qui a été rapporté; ils [ne] peuvent pas être laissés seuls face à ce trauma.» Ainsi, la solitude vécue par Thalia fait écho au processus mortifère de désobjectalisation propre au fonctionnement traumatique. L'aidante vit, en miroir de l'expérience de ses patients, une forme de perte de son sentiment de continuité d'être, étant donné l'absence d'objets symbolisants et contenants pouvant habituellement pallier ce type de ruptures. Elle rapporte que «[les patients] sont aussi seuls quelque part, parce qu'ils ont perdu plein de choses. Ils ont perdu leur maison, leur famille... donc eux aussi se retrouvent face à cette solitude et donc face à un désastre».

Thalia insiste aussi sur l'importance de comprendre ses limites et de demander un soutien professionnel. Elle réitère: «face à ces expériences très difficiles, on ne peut pas être laissés seuls. Il faut quelque part un autre [...] 
pour nous contenir». Elle ajoute qu'une thérapie personnelle ou un travail en binôme peut aussi permettre aux soignants de se protéger de la traumatisation, du fait que ces dispositifs mènent à la diffusion de la charge quantitative ressentie et à la possibilité de "réfléchir à deux». Or, Thalia rapporte avoir souffert d'un traumatisme secondaire qu'elle relie à sa première expérience de soin. Elle dit avoir vu son estime de soi s'affaiblir en raison, peut-être, de son sentiment d'avoir échoué à la tâche impossible qu'est de donner un sens par elle-même à son propre vécu traumatique et à celui de ses patients. Elle évoque un «trauma entendu, un trauma dans l'écoute professionnelle» et aussi, pour elle, paradoxalement, un trauma non-entendu par un autre-sujet. Travailler en contexte humanitaire, c'est «ressentir la destruction et l'accepter; la porter, la contenir pour reconstruire quelque chose avec soi-même et avec l'autre», dit-elle. Mais qui donc fait ce même travail avec elle? Nous notons que Thalia a quitté un de ses milieux de travail en raison de la pauvreté de la qualité des réponses de l'institution à ses demandes de contenance; elle a su amoindrir l'effet traumatique de son expérience en réduisant la durée de son exposition à la situation extrême.

\section{Une demande de soutien à l'appropriation subjective}

Natalie exprime une demande de soutien à l'élaboration qu'elle relie à son sentiment de s'être «déconnectée» ou «détachée» de son expérience d'aide humanitaire. Elle dénonce le fait qu' «il [n'] y a personne qui soit intéressé à [les] préparer à aller là ». Elle n’a reçu aucune formation spécifique à l'intervention en contexte humanitaire avant ou pendant ses missions. Elle ajoute s'être "coupée» de son expérience au moment de son intervention et avoir senti ses symptômes éclore en "après-coup ", "plusieurs mois après ", alors qu'il «n'y avait plus personne» pour l'aider à contenir et penser son vécu. À son arrivée au Québec, suivant sa mission au Kosovo en partenariat avec le gouvernement canadien, elle relate ne jamais s'être fait offrir de suivi psychothérapeutique. Natalie déplore:

Je trouve que c'est une lacune de ne pas avoir fait de suivi par rapport à l'état des employés qui peuvent avoir été dans des situations comme ça. [...] Ces expériences-là, elles méritent d'avoir un débriefing quand on revient. Je trouve que c'est important... Quelques moments, quelques heures de débriefing, et d'orienter après pour être capable de digérer cette expérience-là. 
Natalie exprime de la colère et de la frustration à l'égard des institutions qui omettent de soutenir les aidants humanitaires. Elle sent que cette absence de soutien les empêche de "parler ouvertement» de cette expérience «incompréhensible»; cela les prive d'un lien qui saurait les aider à «s'approprier» ou «faire du sens» de leur vécu. Nous croyons que cela a participé à la mise en place, chez elle, d'un fonctionnement traumatique. Il est à noter que Natalie avait entamé, de son propre gré, un suivi psychothérapeutique, quelques années après son retour. À la fin des entretiens de recherche, elle a avoué avoir su tirer avantage de la possibilité de réaliser une exploration active de cette expérience, toujours en attente de sens. Elle raconte:

Pour moi c'était aidant d'avoir cet espace-là [les entretiens de recherche] pour discuter, pour que ça fasse du sens. [...] Moi je repars avec ça. Avoir eu cet espace-là pour faire du sens de ces expériences-là qui ont été positives et négatives et d'en sortir avec... De me les approprier, plus que tout.

La demande de soutien des participantes est donc élaborée en fonction de leur expérience de soin et de la qualité des réponses de l'objet-environnement à leurs besoins. Elles présentent chacune des réactions psychiques idiosyncrasiques à caractère plus ou moins traumatique. En ce sens, afin que leurs appels ne restent pas sans réponses, nous avons le devoir de penser des dispositifs de métacontenance sensibles et ajustés à leurs réalités. Afin de préserver l'intégrité des soignants, les institutions ont la responsabilité d'offrir des espaces humanisants et subjectivants de contenance pour favoriser l'appropriation subjective et, peut-être, prévenir la cristallisation d'un fonctionnement traumatique. Actuellement, les métacadres (El Husseini, 2017) mis en place par ces institutions sont des plus variables et cela nous mène à nous demander comment les aidantes aménagent leur psyché pour survivre à ces situations extrêmes et à la relation intense qu' elles nouent avec leurs patients.

\section{Les aménagements psychiques des aidantes Une logique d'expulsion et de décharge de la quantité}

Les soignantes ont recours à des processus défensifs visant à décharger, «évacuer» ou «situer à l'extérieur» leur propre trop-plein d'excitation, expérience caractéristique du traumatisme psychique. Ce qu'elles tentent d'expulser, ce sont les contenus mal symbolisés, primaires ou bruts; c'est la destructivité, les pertes et les horreurs qui les habitent; c'est aussi le 
surplus d'angoisse dont elles font l'expérience. Cependant, ce mécanisme peut n'avoir dans certains cas qu'une fonction évacuatrice, alors que dans d'autres, il peut s'avérer une tentative paradoxale d'appropriation subjective ou de remise en fonction de leurs capacités de symbolisation. Marwa raconte:

J'ai besoin d'évacuer, mais d'évacuer sur papier de manière directe, quasiment directe. Le soir même, j'ai besoin d'écrire ce que je ressens, ce que j'ai vécu, même si je ne le relis jamais. Ça, je le ne relis pas, mais je le garde toujours. Je me dis que je sais pas si un jour je vais en avoir besoin...

Marwa emploie l'expression «évacuer de manière directe» lorsqu'elle parle de son besoin de mettre sur papier ses ressentis corporels. On remarque le besoin de décharge de cette aidante, elle-même aux prises avec une intensité intolérable pour ses capacités psychiques. L'expression «je ne les relis pas, mais je les garde toujours » rend compte du paradoxe de devoir expulser un ressenti, mais en même temps de ne pas se couper complètement d'une partie de soi; comme une tentative paradoxale d'appropriation par l'expulsion. Cela illustre combien l'appareil à penser est débordé quantitativement. Marwa précise: «Parfois je n'ai juste pas envie d'entendre quoi que ce soit. Ce n'est pas que je n'ai plus envie d'entendre ces personnes, c'est que j'ai juste envie de n'entendre rien.» Elle raconte comment elle n'a plus les capacités de contenir davantage le vécu des gens qu'elle aide. "À la fin de la journée, après six heures ou sept heures de travail à entendre au moins douze personnes dont sept, par exemple, ont été arrêtées plusieurs fois, torturées, la fatigue est là», précise-t-elle. Le contenant est plein; il ne reste ainsi qu’à «vider son sac» avant de pouvoir se montrer psychiquement disponible de nouveau. Thalia rapporte elle aussi s'être sentie «surchargée», «remplie de pertes», «trop empathique» et, conséquemment, pour survivre à son expérience de soin, avoir dû se trouver un lieu où il était possible de se «vider verbalement ».

\section{S'amputer pour survivre}

Pour illustrer comment le sujet traumatisé en vient à s'amputer d'une partie de soi pour survivre à la situation extrême de la subjectivité ${ }^{3}$, Roussillon (2005) emploie la métaphore du lézard qui, lorsque pris au piège, accepte de sacrifier une partie (sa queue) pour sauver le tout. Tout comme ce reptile, l'individu aux prises avec une souffrance hors de l'entendement se 
dissocie de la partie de son être qui est en contact avec la douleur insupportable pour survivre aux circonstances extrêmes. Ce mode défensif est paradoxal puisqu'il permet la survie de l'appareil psychique au coût de l'intégrité du Moi, qui se voit clivé et privé d'une sphère de son expérience. Il permet par ailleurs de renforcer le sentiment de maitrise du sujet sur la situation, en lui permettant de passer d'un mode "passif», où la souffrance est subie, à un mode "actif» où il a au moins l'illusion de la contrôler. Natalie semble être la participante qui a mis en place un système défensif correspondant à ce type de logique désubjectivante. L'aidante a entamé le processus d'entretiens de recherche en insistant sur son sentiment d'étrangeté par rapport à son expérience. Lors de notre rencontre initiale, elle indique: «C'est comme si c'était une autre moi qui était allée [à l'étranger].» À de nombreuses reprises, elle décrit son impression de s'être "détachée» d'une partie d'elle pour continuer son travail en contexte humanitaire. Elle dit: «Je pense que quand tu fais ce travail-là, il faut que tu te coupes un peu. Moi mon expérience, c'est que je me suis coupée pour être capable de rester là, parce que c'était dangereux.»

Natalie a dû effectuer une scission dans sa subjectivité et cela semble avoir ébranlé sa capacité à se représenter et s'approprier ce qu'elle a vécu, en plus de désaffecter son expérience (McDougall, 1989). L'aidante rapporte ne pas avoir bénéficié d'un soutien pour « digérer» ou «s'approprier» son expérience d'intervention, c'est-à-dire pour parvenir à la "garder audedans» de façon à la transformer plutôt que devoir la mettre hors de la psyché pour ne pas en être malade. Natalie évoque son sentiment d'être « déconnectée» de ce qu'elle a vécu. «Je ne me souviens pas d'avoir pleuré, je ne me souviens pas d'avoir été si connectée que ça», soulève-t-elle. L'aidante raconte: «J'étais vraiment déconnectée. Pourquoi? Je pense que je n'aurais pas résisté. Je ne serais pas restée là. J'aurais eu peur. Je l'ai [son vécu affectif] mis dans une petite case en dedans de moi et j'ai fermé ça.» Elle décrit s'être coupée d'une partie d'elle, car elle croit qu'elle n'y aurait pas survécu psychiquement. Nous observons comment cette participante s'est protégée de façon à «ne pas sentir» la "peur» et les affects déstructurants associés à son expérience de soin.

L'ensemble de ces processus défensifs s'inscrit dans un continuum en rapport aux coûts qu'ils engendrent pour l'appareil psychique et à l'implication de l'objet dans l'abaissement des tensions. Lorsque les soignantes en viennent à faire elles-mêmes l'expérience d'une surcharge de leurs capacités moïques et à se voir habitées de contenus mal symbolisés, cela les contraint 
à adopter un mode défensif de survivance psychique. Elles expulsent sans possibilité d'appropriation subjective ou, de manière plus radicale, se mutilent d'une sphère complète de leur expérience pour préserver leur intégrité. Toutefois, lorsque les conditions environnementales ne sont pas aussi extrêmes, sur le même registre, il leur est aussi possible d'effectuer une «coupure» symbolique en quittant les lieux d'intervention ou en laissant libre cours à leurs pulsions de vie. D'autres processus, plus rarement observés, n'impliquent ni une coupure d'une partie de soi, ni une position de repli relationnel, mais, au contraire, permettent un réinvestissement libidinal dans un contexte relationnel. Par ailleurs, lorsque ces processus défensifs échouent à juguler l'angoisse, on observe l'éclosion d'une multitude de symptômes tels que la dépression, la déflation narcissique, la reviviscence et l'hypervigilance.

\section{Propositions de dispositifs métacontenants}

Il est possible d'imaginer des mesures qui pourraient permettre de mieux préparer, soutenir et contenir les aidants humanitaires face aux phénomènes traumatiques. Quelques auteurs ont déjà soulevé succinctement qu'il était important de bien former et sensibiliser les soignants aux multiples enjeux de l'intervention en contexte humanitaire. D'autres ont exposé la nécessité d'avoir recours à de la supervision ou à des groupes d'inter-vision. D'autres encore ont souligné l'importance d'offrir des espaces de psychothérapie aux soignants, en plus de les encourager à diversifier leurs activités professionnelles et personnelles (Ladislav Paviae, 1999; Delbrouck et al., 2011; Petiau, 2016; Tarazi-Sahab, El Husseini et Moro, 2016; Cusin et Fabre, 2017; El Husseini, 2017; Karray et al., 2017). Nous croyons de notre côté pouvoir proposer un type de soutien simple et adapté aux difficultés spécifiques que nous avons identifiées, à savoir notamment la difficulté de contenance, la difficulté de symbolisation de l'expérience extrême et le risque d'amputation d'une partie de soi.

\section{Le préalable}

Si l'impréparation au danger est un élément causal important des réactions traumatiques (Freud, 1920), il est logique de penser que la formation des aidants humanitaires devrait tenter de les préparer non seulement sur le plan technique, mais aussi et surtout sur le plan psychique. En plus des connaissances habituelles sur le milieu (sa culture, son histoire, etc.) et sur les tâches attendues, les aidants ont avantage à avoir une compréhension 
suffisante des difficultés psychiques et relationnelles qu'ils rencontreront. Une telle connaissance, à elle seule, ne peut prévenir avec certitude les réactions que nous avons identifiées, mais nous savons que l'impréparation et le manque de contre-investissement jouent un rôle important dans les réactions traumatiques (Freud, 1920); la représentation des difficultés traumatiques prévisibles pourrait, dans certains cas, en atténuer la violence et la soudaineté. Les soignants gagneraient aussi à être sensibilisés aux effets de l'identification empathique aux victimes de traumatismes et à la nécessité de trouver pour eux-mêmes un lieu d'écoute et de contenance.

\section{Contenir le conteneur durant l'expérience d'aidant}

Le paradoxe soulevé par cette étude est que les aidantes doivent offrir une écoute empathique et une contenance aux gens traumatisés qu'elles soignent, mais que cette position les met à risque de vivre des impasses psychiques similaires limitant leurs propres capacités de symbolisation. Si leur rôle est de contenir et d'aider à l'élaboration psychique, qui les aide pour ce même travail? Nous savons à quel point, quand l'appareil psychique est débordé, l'individu a besoin d'un autre semblable pour retrouver ses capacités (Roussillon, 2012). D’ailleurs, ces aidantes se sont plaintes de ne pas avoir de lieu pour exprimer leurs difficultés et se sentir contenues.

Cette fonction métacontenante peut prendre plusieurs formes. Il ne s'agit pas d'offrir une psychothérapie au sens strict du terme, bien que certains puissent en sentir le besoin. Il ne s'agit ni d'une approche visant à nier ou remplacer des vécus pénibles par des cognitions calmantes ni d'une approche recherchant à identifier des sources personnelles de vulnérabilité, mais il s'agit plutôt de fournir la possibilité aux aidants d'avoir quelqu'un qui puisse écouter et comprendre les émois et le désespoir. Souvent, leurs difficultés font en sorte que leurs vécus ou leurs angoisses constituent des «messages» qui n'arrivent pas à se formuler ou se signifier. L'objectif est de remettre en marche la capacité de penser ces ressentis en se sortant peu à peu du fonctionnement hallucinatoire (Reid, 2008) qui les amène à vouloir s'amputer de ces émois pénibles. Il s'agit d'une écoute réflexive où un «autre semblable» se laisse toucher et tente d'effectuer une élaboration des ressentis dans un processus de pensée partagée (Roussillon, 2008). Il s'agit donc de com-prendre: prendre avec, ressentir avec l'autre et penser avec l'autre semblable. Cela peut se faire dans un espace de supervision ou d'inter-vision exempt de toute dimension de "contrôle» ou d'évaluation de l'aidant; l'aidant doit pouvoir, en toute confiance, mettre en jeu et tenter de 
communiquer autant ce qu'il arrive à représenter de ses difficultés que ce qu'il peine à comprendre et à identifier. Il n'est pas facile dans les milieux où ouvrent ces soignants de mettre en place un tel espace, mais certains nous ont mentionné comment l'utilisation d'une technologie comme Skype pouvait leur permettre de parler à quelqu'un de confiance qui remplissait cette fonction.

\section{Au retour, une latence et un temps d'appropriation}

Le retour d'une mission humanitaire ne signifie pas nécessairement le retour à l'équilibre psychique ou à soi-même. Les soignants rapportent avoir besoin de «se retrouver» lors de leur retour au pays, c'est-à-dire retrouver la personne qu'ils étaient avant, y compris la personne capable d'affects normaux. Or, les défenses mobilisées (désaffectation, autotomie, décharge), bien qu'elles puissent s'estomper, peuvent aussi enrayer de façon quasi permanente ce processus dans une tentative inconsciente d'empêcher la reviviscence de l'effroi. Ainsi, comment «se retrouver» quand on s'est départi d'une partie de soi? Certains aidants sentent le besoin d'entreprendre un processus psychothérapique et s'y investissent par leurs propres moyens. Mais ne pourrait-on pas proposer de façon plus systématique un dispositif, non pas psychothérapique au sens strict, mais offrant l'occasion d'un «dégel» psychique, d'une reprise de la symbolisation et de l'appropriation subjective de leur expérience?

Comme certaines des aidantes que nous avons rencontrées avaient trouvé spontanément des modes de reprise de la symbolisation, nous nous sommes inspirés de leurs besoins pour imaginer des ateliers utilisant des médiations thérapeutiques comme l'écriture, le dessin ou le psychodrame. Une des fonctions de ces médiations thérapeutiques serait la reprise du travail de la symbolisation et ultimement de l'appropriation subjective de leur expérience traumatique. Ces ateliers, en petits groupes, ne viseraient pas essentiellement l'interprétation d'un conflit ou d'un contenu inconscient, bien qu'elle puisse advenir dans certaines circonstances. Ils auraient comme objectif le soutien à l'élaboration psychique et à la symbolisation et se centreraient sur trois «lieux» de représentations. Le premier lieu de travail est celui de la potentialité de représentation de ce qui a été traumatique, de ce qui a fait effraction dans l'appareil psychique. Le deuxième serait celui du potentiel de représentation du processus traumatique lui-même (comme les processus de sidération, de gel des émotions, d'amputation psychique). Le troisième serait celui de la représentation de l'objet qui «pourrait» 
potentiellement remplir des fonctions de contenance et de symbolisation; fonctions qui ont pu faire défaut lors d'un travail humanitaire traumatisant. Autrement dit, il s'agit d'utiliser les médiations dans leur potentiel de représentation non seulement des contenus, mais surtout des processus en cause et des fonctions attendues de l'objet, qui d'ailleurs ont peut-être fait défaut.

\section{Conclusion}

Cet article se veut une réflexion sur divers éléments propres au vécu particulier du soin en contexte humanitaire, mais aussi une mise en lumière du manque de soutien de ces soignantes, déployées sans égard à la préservation de leur propre intégrité. Ces dernières sont confrontées à des conditions insensées et désubjectivantes dans l'exercice de leur profession. Elles sont appelées à capter et transformer le vécu brut et chargé quantitativement des sujets qu'elles prennent en charge. Ce phénomène les mène à faire l'expérience en miroir de vécus semblables à ceux de leurs patients, mettant à rude épreuve leurs capacités de contenance et de symbolisation et diminuant leur disponibilité psychique.

Nous exposons comment, en réponse au déséquilibre psychique vécu par les aidantes, elles formulent de façon plus ou moins élaborée une demande de métacontenance: le contenant a lui aussi besoin d'être contenu pour continuer à remplir sa fonction et pour ne pas verser lui-même dans une réaction traumatique.

Devant la qualité de la réponse de l'objet-environnement à ce besoin de métacontenance, les soignantes présentent différentes solutions psychiques plus ou moins couteuses pour l'économie de leur psyché. Confrontées à l'angoisse que génèrent les situations extrêmes de la subjectivité, elles érigent un système défensif de survivance psychique qui peut aller jusqu'à l'amputation d'une partie de soi. Lorsque le système défensif est mis en échec et que la barrière de pare-excitation de la psyché est fissurée, les soignantes peuvent faire l'expérience d'un traumatisme secondaire. Nous entendons par cela l'éclosion d'un type de fonctionnement en traumatique ainsi que l'émergence d'une panoplie de symptômes.

À la lumière des expériences de désubjectivation vécues par les aidantes, nous croyons nécessaire que les institutions mettent en place un cadre métacontenant et symboligène au sein de leurs organisations. Au vu des solutions couteuses de coupure de soi et de sidération que nous avons décrites, nous croyons qu'il faille offrir un lieu et une écoute permettant la remise en 
marche des capacités de penser et de symboliser les ressentis traumatiques. Il s'agit d'une écoute réflexive où un «autre semblable» peut être touché et où il est possible d'effectuer une certaine élaboration des émois dans un type de pensée partagée (Roussillon, 2008) et même de jeu créatif.

\author{
David Smolak \\ david.smolak.1@gmail.com \\ Louis Brunet \\ brunet.louis@uqam.ca
}

\title{
Notes
}

1. Type de fonctionnement psychique où l'économie de l'appareil à penser s'organise en fonction de la compulsion de répétition et des tentatives de représentation, de figuration ou de symbolisation des effets du traumatisme.

2. Des prénoms fictifs sont employés et certaines données biographiques ont été modifiées ou consciemment omises afin de préserver l'anonymat et la sécurité des participantes.

3. Circonstances «dans lesquelles la possibilité de continuer de se sentir "sujet", de continuer de maintenir le sentiment de son identité, et d'une identité inscrite au sein de l'humaine condition, est portée à son extrême, voire au-delà du pensable» (Roussillon, 2005, p. 221)

\section{Références}

Bettelheim, B. (1960). Le coeur conscient. Paris: Laffont, 1972.

Bion, W. R. (1962). Une théorie de l'activité de pensée. Dans Réflexion faite. Paris: Presses universitaires de France, 1983.

Bokanowski, T. (2002). Traumatisme, traumatique, trauma. Revue française de psychanalyse, 66, 745-757. doi: 10,391 7/rfp.663.0745

Bokanowski, T. (2010). Du traumatisme au trauma: les déclinaisons du traumatisme en psychanalyse. Psychologie clinique et projective, 16, 9-27.

Brunet, L. (2000). L'identification projective et la fonction contenante: illusions nécessaires ou délire partagé? Journal de la psychanalyse de l'enfant, 26, 161-192.

Brunet, L. et Casoni, D. (2000). A Necessary Illusion: Projective Identification and the Containing Function. Klein on Projective Identification. Canadian Journal of Psychoanalysis, 9, 137-163

Connorton, E., Perry, M. J., Hemenway, D. et Miller, M. (2012). Humanitarian relief workers and trauma-related mental illness. Epidemiologic reviews, 34(1), 145-55. doi: 10,1093/ epirev/mxr026

Cusin, J. et Fabre, C. (2017). Proposition d'un modèle conceptuel du traumatisme vicariant appliqué à la gestion des ressources humaines: le cas des conseillers en accompagnement vers l'emploi. Revue de gestion des ressources humaines, 104, 3-18.

Delbrouck, M., Venara, P., Goulet, F. et Ladouceur, R. (2011). Syndromes connexes au burnout du soignant. Dans Comment traiter le burn-out (p. 289-303). Paris: De Boeck.

El Husseini, M. (2017). Réactions contre-transférentielles en terrain de guerre. Extrait d'une recherche sur l'exploration du contre-transfert dans la clinique du trauma. Enfances \& Psy, 74, 43-50. 
Ferenczi, S. (1932). Journal clinique. Paris: Payot, 1986.

Freud, S. (1920). Au-delà du principe de plaisir. Dans Euvres complètes, vol. XV (p. 273 228). Paris: Presses universitaires de France, 1996.

Green, A. (1990). La folie privée. Paris: Gallimard.

Karray, M., Cénat, J. M., Derivois, D., Anaut, M. et Jacome, M.-C. (2017). Soigner aux frontières: regard psychodynamique sur le quotidien traumatique des soignants/réfugiés. Revue québécoise de psychologie, 38 (3), 79-98.

Ladislav Paviae, E. K. (1999). Countertransference and Empathic Problems in Therapists/ Helpers Working with psychotraumatized Persons. Croatian Medical Journal - Clinical Medecine, 40(4), 466-472.

Milner, M. (1952-1954). Le rôle de l'illusion dans la formation du symbole. Dans B. Chouvier (dir.), Matières à symbolisation. Art, création et psychanalyse (p. 49-51). Lausanne: Delachaux et Niestlé (1998).

McDougall, J. (1989). Théâtres du corps. Le psychosoma en psychanalyse. Paris: Gallimard.

Ottino, S. von O. (2007). Violences extrêmes: le poids de la réalité à l'épreuve de la causalité psychique. Psychothérapies, 27, 127-138.

Petiau, A. (2016). Ne dites surtout pas que vous êtes médecins. Cahiers critiques de thérapie familiale et de pratiques de réseaux, 57, 103-118.

Quosh, C., Eloul, L. et Ajlani, R. (2013). Mental health of refugees and displaced persons in Syria and surrounding countries: a systematic review. Intervention, 11 (3), 276-294.

Reid, W. (2008). Un nouveau regard sur la pulsion, le trauma et la méthode analytique. Première partie: une théorie de la psyché. Filigrane, 17 (1), 68-94.

Roussillon, R. (2000). Traumatisme et liaisons primaires non symboliques, Actualités psychosomatiques, 3, 89-109.

Roussillon, R. (2002). Jalons et repères de la théorie psychanalytique du traumatisme psychique. Revue belge de psychanalyse, 40, 25-42.

Roussillon, R. (2005). Les situations extrêmes et la clinique de la survivance psychique. Dans J. Furtos et Ch. Laval (dir.), La santé mentale en actes. De la clinique au politique (p. 221238). Toulouse: Éditions Érès.

Roussillon, R. (2008). Le transitionnel, le sexuel et la réflexivité. Paris: Dunod.

Roussillon, R. (2012). On souffre du non approprié de l'histoire: on guérit en l'intégrant. Le carnet psy, 9 (167), 36-41.

Roussillon, R. (2014). Manuel de pratique clinique. Paris: Elsevier Masson.

Smolak, D. et Brunet, L. (2017). Interprétations psychanalytiques du traumatisme: une synthèse théorico-clinique. Revue québécoise de psychologie, 38 (3), 99-124.

Tarazi-Sahab, L., El Husseini, M. et Moro, M. R. (2016). L'accueil de patients traumatisés: la langue maternelle, un levier thérapeutique médiatisant? Cliniques, 11, 72-88.

Winnicott, D. (1971). Jeu et réalité. Paris: Gallimard. 